Disponível em

http://www.anpad.org.br/rac

RAC, Rio de Janeiro, v. 18, n. 4, art. 6, pp. 487-507, Jul./Ago. 2014

http://dx.doi.org/10.1590/1982-7849rac20141722

$($ co) EY-No

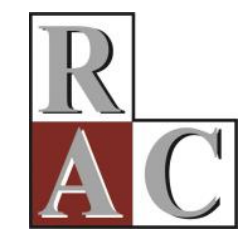

\title{
Ações Coletivas e Tecnologia da Informação: Efeitos Indutores à Configuração dos Coletivos Inteligentes
}

\section{Collective Action and Information Technology: Stimulating Configuration of Intelligent Collectives}

Jefferson David Araujo Sales E-mail: profsales@hotmail.com Universidade Federal de Sergipe - UFS Av. Marechal Rondon, s/n Jardim Rosa Elze, CCSA 2, S1. 13, 49100-000, São Cristóvão, SE, Brasil.

Jairo Simião Dornelas E-mail: jerh57@gmail.com Universidade Federal de Pernambuco - UFPE Av. dos Economistas S/N, CCSA, Cidade Universitária, 50670-902, Recife, PE, Brasil.

Artigo recebido em 06.11.2013. Última versão recebida em 25.03.2014. Aprovado em 30.03.2014. 


\title{
Resumo
}

Comunidades de prática $(\mathrm{CoP})$ são contextos propícios para o surgimento de coletivos inteligentes, que são espaços voltados para a interação. O caso evidenciado neste estudo exemplifica como o Conselho Nacional das Entidades de Provedores de Serviços de Internet (CONAPSI) apresenta aspectos similares aos das CoP e, logo, desenhou-se como objetivo norteador da investigação analisar como as ações coletivas suportadas pela tecnologia da informação caracterizam-se como elementos indutores à ascensão das CoP a coletivos inteligentes. Para dar suporte ao estudo de caso, a fundamentação teórica trouxe definições e características das comunidades de prática, dos coletivos inteligentes e das tecnologias da informação que apoiam grupos. $\mathrm{Na}$ investigação, foram empreendidas análises documentais em listas de discussão eletrônicas, tratadas com estatística descritiva, e em entrevistas com representantes do Conselho, interpretadas com análise de conteúdo. Os resultados demonstram que o uso de listas de discussão eletrônicas impulsiona o surgimento de espaços virtuais de convivência e, consequentemente, coletivos inteligentes virtuais.

Palavras-chave: coletivos inteligentes; comunidades de prática; tecnologias colaborativas.

\begin{abstract}
Communities of Practice (CoP) are appropriate settings for the emergence of intelligent collective spaces that are meant for interaction contexts. The case shown in this study exemplifies how the National Council of Internet Service Provider Entities (CONAPSI) has similar aspects to a CoP. This soon found a guiding purpose of analyzing how collective actions supported by information technology are characterized as inducing CoPs to become intelligent collectives. To support the case study, the theoretical foundation included definitions and characteristics of communities of practice, intelligent communities and information technologies that support groups. Research consisted of conducting document analysis of online discussion threads, treated with descriptive statistics, and interviews with Council representatives, interpreted using content analysis. Results demonstrate that the use of electronic discussion drives the emergence of virtual living spaces and consequently intelligent virtual communities.
\end{abstract}

Key words: collaborative technologies; communities of practice; intelligent collectives. 


\section{Introdução}

Desde a década de 1990, temas voltados à colaboração, cooperação, coletividade e ao compartilhamento entraram nas pautas de discussões dos acadêmicos e das organizações. Inteligência coletiva (Lévy, 1994), gestão do conhecimento (Nonaka \& Takeuchi, 1997), intercriatividade (BernersLee, 2000), inteligência emergente (Johnson, 2001) e coletivos inteligentes (Costa, 2008; Laboratório de Estudos em Inteligência Coletiva e Biopolítica [LINC], 2008; Rheingold, 2002) representam algumas das tentativas de se pôr em discussão a questão da construção coletiva do conhecimento nas organizações, assim, reedificando o valor do trabalho em grupo.

A seu turno, as organizações modernas têm, justamente, como pressuposto básico dividir o trabalho entre os seus grupos (Dubrin, 2003; Robbins, 2013), pois esses canalizam os esforços coletivos em prol de um objetivo comum. Nessas circunstâncias, é corriqueiro encontrar grupos de trabalho que optem por utilizar ferramentas tecnológicas na execução suas tarefas, para reduzir o tempo de realização, os custos de transação e as distâncias geográficas.

Por permitir a criação de ambientes de colaboração e cooperação nas organizações, a tecnologia da informação apresenta-se como elemento essencial para os grupos e fornece o suporte ao trabalho através de ferramentas de comunicação e de colaboração, bem representadas por tecnologias colaborativas e groupware (Kolfschoten, Niederman, Briggs, \& Vreede, 2012). Todas essas ferramentas tecnológicas possibilitam a realização de atividades conjuntas que envolvem muitas pessoas, as quais não precisam necessariamente estar presentes no mesmo local físico. Entre as diversas formações socioorganizacionais que recebem influências da tecnologia da informação e são voltadas para ações coletivas, estão aquelas que têm como elementos caracterizadores a interação e a troca de conhecimento entre seus membros, como é o caso dos grupos e das comunidades de prática $(\mathrm{CoP})$.

As comunidades de prática podem ser entendidas como grupos de pessoas que buscam desenvolver tarefas em comum, no intuito de criar algo coletivo em um domínio de conhecimento vinculado a uma determinada prática (Souza-Silva \& Davel, 2007; Wenger, 2003; Wenger, White, \& Smith, n. d.). Já na década de 1990, ao eleger as características principais das comunidades de prática, Wenger (1998) menciona o fato de que elas proporcionam a oportunidade de seus componentes desenvolverem capacidades e conhecimentos através do intercâmbio de experiências, sendo a TI um elemento propulsor dessas atividades, forjando menções à inteligência em coletivos.

O termo inteligência coletiva (IC), que neste artigo dá subsídio para os coletivos inteligentes, foi utilizado pela primeira vez em estudos acadêmicos, até onde se pôde apurar, em 1976, em trabalhos de Murray Turoff (Rheingold, 1996). Tais estudos objetivavam difundir a ideia de que as interconexões entre computadores proporcionariam atividades coletivas e, consequentemente, um saber que poderia ser coletivizado. Já Lévy (1994) deu visibilidade ao termo ao mencionar que a IC poderia ser entendida como sinônimo de um saber comunitário inserido num cyberespaço.

Seguindo essa mesma corrente de pensamento, Rheingold (2002) desenvolveu a ideia dos coletivos inteligentes (CI), que foram definidos como um conjunto de ações coletivas que podem acontecer em grupos. Conforme esse autor, no suporte às ações desses coletivos inteligentes está a tecnologia da informação calcada em atributos como fácil usabilidade, habilitação para conexões e abertura às interações.

O Conselho Nacional das Entidades de Provedores de Serviços de Internet (CONAPSI), caso central desta investigação, tem suas principais atividades voltadas para a defesa dos interesses da classe por ele representada, ratificando, pois, que um dos motivos que conduzem as empresas a unirem-se em associações é a possibilidade da ação coletiva, já que seus integrantes são representados de forma única junto a governos, entidades classistas e mercados (Serviço Brasileiro de Apoio às Micro e Pequenas Empresas [SEBRAE], 2009). 
O ramo dos provedores de internet no Brasil está constituído, em sua esmagadora maioria, por empresas de pequeno porte, que veem nas associações possibilidades de criação de redes de interação, como as comunidades de prática, para que possam gerar novos conhecimentos e concorrer com os grandes grupos do setor. Assim, é relevante ter o CONAPSI no centro desta pesquisa, já que ele engloba $80 \%$ das empresas de provedores de Internet (ANATEL, 2011), sendo, então, bastante representativo o seu estudo.

Ao se atinar para o cenário apresentado, percebe-se que, mesmo com grandes predisposições para situações de coletividade, as comunidades de prática necessitam de contextos organizacionais voltados para o surgimento de ambientes colaborativos (Schommer, 2005). É preciso que surjam condições próprias que levem as pessoas a quererem se engajar verdadeiramente neste tipo de estrutura social. Mas, devido a aspectos ainda pouco conhecidos, acredita-se que tais condições nem sempre estejam disponíveis.

Somando-se a isso, surgem questões voltadas para o uso de tecnologias apropriadas para as necessidades das CoP (Wenger et al., n.d.). Entre essas, aquelas que objetivam dar suporte às ações de colaboração e cooperação são cada vez mais comuns, porém nem sempre são utilizadas adequadamente. Assim, é de se questionar como as comunidades de prática utilizam as tecnologias da informação que estão à sua disposição.

Tendo como tema norteador a coletividade suportada por aparatos tecnológicos, configura-se, então, como objetivo central desta investigação, a análise, sob a luz da tecnologia da informação, das ações de colaboração e cooperação em comunidades de prática que surgem em associações de empresas provedoras de internet, mais especificamente no CONAPSI, em busca de efeitos caracterizadores de coletivos inteligentes.

A intenção maior do estudo é contribuir para o entendimento da realidade das comunidades de prática e de sua configuração para coletivos inteligentes. Os seus resultados buscam o aprofundamento das discussões com o intuito de trazer novas evidências para a literatura das CoP, em especial, pela incorporação de aspectos expandidos para a realidade do setor evidenciado no estudo e também TI e aspectos organizacionais.

\section{Fundamentação Teórica}

Esta seção trata de apresentar os conceitos centrais que fundamentam o estudo. Tem-se como marco inicial o ambiente organizacional povoado por grupos de pessoas, os quais, quando organizados e obedecendo a certos atributos, podem ser percebidos como comunidades de prática. A busca é fundamentar que, principalmente na virtualidade, os coletivos inteligentes surgem quando as ações coletivas são impactadas pelo uso da tecnologia da informação, mormente, aquelas voltadas para os grupos.

\section{Comunidades de prática}

A literatura atinente à abordagem socioprática (Araujo, 1998; Argote, 2005; Gherardi \& Nicolini, 2000; Simpson, 2011; Wenger, 1998; Wenger, Mcdermott, \& Snyder, 2002) vem gerando, nos últimos anos, compreensões importantes para os fenômenos organizacionais, como é o caso das comunidades de prática. Isso acontece porque a componente base para tal abordagem é a interação social, que provoca, como resultado, o engajamento das pessoas em grupos e comunidades (Gazzoli, 2012; Maria, Faria, \& Amorim, 2008). Logo, a noção de comunidade de prática (CoP) emerge, aqui, por considerar a interação como a ação social ideal para a tentativa de representar os processos de geração de conhecimento nos níveis individual e organizacional (Easterby-Smith, Crossan, \& Nicolini, 2000; Souza-Silva, 2009; Souza-Silva \& Davel, 2007). 
Quando surge um contexto no qual pessoas geram conhecimento juntas, surge também uma comunidade de prática (Wenger, 2001), com o fundamento de que "todos nós pertencemos a comunidades de prática" (p. 3) e que "de fato, as comunidades de prática estão por todas as partes" (p. 3 ), pois as pessoas filiam-se a elas no trabalho, em casa, na escola e em todos os lugares que frequentam.

Assim, o grupo de pessoas que compartilha algum interesse em comum a partir do domínio de atividades, ou tarefas específicas, e que, neste cenário, dê ênfase aos processos de desenvolvimento coletivo, é tido como uma comunidade de prática (Brandon \& Charlton, 2011; Wenger, 2001).

Advoga-se que o núcleo central das organizações continua sendo o conjunto de processos de negócio e a busca por resultados, mas parece certo que as comunidades de prática geram um impacto nas estruturas organizacionais, adicionando-lhes uma camada de complexidade, que é uma forma de estrutura ortogonal centrada no conhecimento (Brandon \& Charlton, 2011; Wenger, 2001), não significando, necessariamente, a reestruturação de todo o sistema organizacional.

Salienta-se que a adesão das pessoas a tais comunidades acontece de maneira informal, voluntária e espontânea (Souza-Silva \& Davel, 2007). Não existe a preocupação de seguir padrões de conformidade, como a estrutura formal de uma organização, por exemplo (Habhab-Rave, 2010).

Mesmo sendo vista como uma estrutura informal, que age de maneira paralela à organização, a comunidade de prática, de acordo com a literatura, possui fortes laços com a estrutura formal por conta da maneira como se apresenta o engajamento dos seus membros.

Estes membros são também profissionais com atribuições definidas na estrutura formal e que partilham entre si experiências e conhecimentos ligados às práticas profissionais em um multiassociativismo. A Figura 1 esboça o que Wenger (2001) taxa como os três aspectos estruturantes das comunidades de prática: o domínio, a comunidade e a prática.

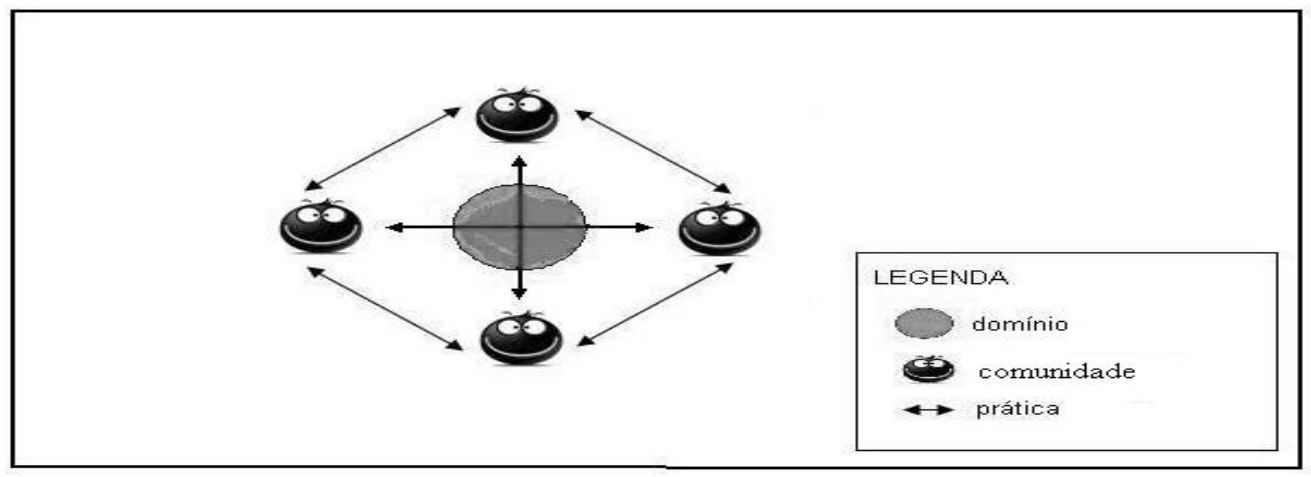

Figura 1. Aspectos Estruturantes das Comunidades de Prática.

Fonte: Baseado em Wenger, E. (2001). Supporting communities of practices a survey of community-oriented technologies: how to make sense of this emerging market understand the potential of technology and set up a community platform. Version 1.3. Recuperado de http://archive2.nmc.org/projects/dkc/Technology_Survey.doc

O domínio pode ser facilmente entendido como o tema central que baliza a existência da comunidade de prática. A adesão à $\mathrm{CoP}$ implica, para o indivíduo, um nível mínimo de conhecimento sobre o domínio em questão. Isso não significa que tal indivíduo deva ter algum conhecimento advindo de experiências práticas fora da comunidade, mas que possua, pelo menos, uma noção elementar do objetivo primeiro, ou principal, da comunidade. Como consequência da existência de um domínio, temse a distinção precisa daqueles que são membros da CoP das outras pessoas que não o são.

Uma CoP necessita ter, ao redor do seu domínio, um conjunto fixo, ou quase fixo, de membros. Esses membros, que compõem essa comunidade, devem estar engajados e participar ativamente de discussões conjuntas, auxiliando uns aos outros e coletivizando informações, construindo, assim, relacionamentos. 
Por fim, para que se configure como uma comunidade de prática, o grupo deve desenvolver ações de compartilhamento de recursos, experiências, ferramentas, técnicas, informações e hábitos de coletivizar conhecimento. Assim, a constância, a repetição e o compromisso em coletivizar informação e conhecimento fazem parte da prática, tornando-a propensa a um efetivo feedback.

\section{Ações e coletivos inteligentes}

A interação entre pessoas é condição básica para a realização do trabalho em equipe e, sobre isso, Lévy (1994) menciona dois aspectos influenciadores: o uso de instrumentos que permitem a enunciação coletiva e as formas de realizar ações em conjunto. O primeiro aspecto influenciador refere-se à tecnologia, em particular, da informação, que estrutura as formas de interação e compartilhamento; e, o segundo, retrata os tipos de ações realizadas pelas pessoas nas organizações.

Entre os tipos de ações supramencionados, destaca-se, em primeiro plano, a colaboração que acontece quando os diversos atores trabalham em conjunto por meio de ajuda mútua, de forma legítima, tendo como fim o alcance de objetivos que beneficiem a todos (Boavida \& Ponte, 2002). Nessas condições, o simples fato de agir em conjunto não significa que as pessoas estão em colaboração, pois é preciso que existam, também, bases de igualdades na realização do trabalho.

Argyle (1991) enxergou a cooperação, que também se caracteriza como um tipo de ação coletiva, como uma ação coordenada que acontece em conjunto, podendo surgir em diversos ambientes como trabalho e relacionamentos sociais, visando atingir objetivos comuns. Para Costa (2008), a cooperação é orientada pela lógica de apropriação de resultados em que, através da realização de tarefas, busca-se atingir os objetivos traçados. Assim como acontece com a colaboração, a cooperação também é uma forma de interação entre as pessoas, mas que se diferencia da primeira por estimular relações interindividuais que resultam em sistemas de interações (Goco, 2006). A Tabela 1, a seguir, faz uma diferenciação entre as duas formas de interação, com o propósito de facilitar o entendimento e estruturar os conceitos.

Tabela 1

\section{Características das Ações de Colaboração e Cooperação}

\begin{tabular}{|c|c|c|c|c|}
\hline Ação coletiva & Núcleo & Contexto & Forma de interação & Características \\
\hline Colaboração & Trabalho & $\begin{array}{l}\text { Conjunto de } \\
\text { atividades }\end{array}$ & $\begin{array}{l}\text { Global, já que as pessoas } \\
\text { interagem em todas as etapas } \\
\text { do trabalho. }\end{array}$ & $\begin{array}{l}\text { É desenvolver em conjunto } \\
\text { atividades de um trabalho e } \\
\text { envolve pensar, refletir, } \\
\text { discutir, preparar, formar e } \\
\text { empenhar-se. Portanto requer } \\
\text { um grande número de } \\
\text { operações. }\end{array}$ \\
\hline Cooperação & Operação & Tarefa & $\begin{array}{l}\text { Local, já que as pessoas } \\
\text { interagem na realização de } \\
\text { uma determinada tarefa. }\end{array}$ & $\begin{array}{l}\text { É a realização de operações } \\
\text { (tarefas) em conjunto, de } \\
\text { forma clara e bem definida. } \\
\text { Como resultado, tem-se o } \\
\text { produzir ou fazer funcionar } \\
\text { algo de acordo com o que foi } \\
\text { planejado. }\end{array}$ \\
\hline
\end{tabular}

Nota. Fonte: Argyle, M. (1991). Cooperation: the basis of sociability. London: Routledge; Boavida, A M., \& Ponte, J. P. (2002). Investigação colaborativa: potencialidades e problemas. In Grupo de Trabalho sobre Investigação (Org.), Refletir e investigar sobre a prática profissional (p. 43-55). Lisboa: APM; Goco, A. L. P. (2006). Cooperação versus colaboração: conceitos para o ensino de enfermagem em ambiente virtual. Revista Brasileira de Enfermagem, 59(5), 680-683. doi: 10.1590/S0034-71672006000500016; Costa, R. da (2008). Inteligência coletiva: comunicação, capitalismo cognitivo e micropolítica. Famecos, 1(37), 61-68. 
A ideia de coletivos inteligentes, ora em construção, é impulsionada pelo entendimento de inteligência coletiva, que, para Lévy (1994, p. 28), é "uma inteligência distribuída por toda parte, incessantemente valorizada, coordenada em tempo real, que resulta em uma mobilização efetiva das competências". Esta definição tem como base uma coletivização inteligente da informação e do conhecimento que visa, por meio da interação, ao enriquecimento mútuo de todas as pessoas envolvidas, sem distinção. Aqui, ficam de fora aquelas comunidades que não têm como prioridade o desenvolvimento de todos os envolvidos.

Para Costa (2008), a inteligência coletiva é sinônimo de criação, circulação e multiplicação de ideias em contextos coletivos. E isso significa que, na prática, a inteligência coletiva trabalha com ideias que circulam em locais comunitários, presenciais ou virtuais e tem como consequências um conhecimento desenvolvido por todos e para todos.

Assim, percebe-se que o termo inteligência coletiva (Lévy, 1994) exerceu influência sobre os trabalhos de Berners-Lee (2000), Johnson (2001), Surowiecki (2004), Romani e Kuklinski (2007), Costa (2008), entre outros, e tem participação importante no entendimento do que sejam os coletivos inteligentes, uma vez que estes podem funcionar como cenário ideal para produção desse tipo de inteligência.

Tomando como base as definições vistas aqui, poder-se-ia conceituar coletivos inteligentes como sendo espaços propícios para a interação através de ações coletivas (colaboração e cooperação) que admitem estruturas presenciais ou virtuais e que utilizam a tecnologia da informação para promover a comunicação e o compartilhamento de conteúdos entre os seus membros. Não há, com isso, intenção de sugerir a criação de mais um tipo de inteligência, mas sim de dar importância a uma construção comunitária ou verdadeiramente coletiva de ideias, conhecimentos e saberes. Nessa perspectiva, é necessário que sejam elaborados e oferecidos meios tecnológicos que façam com que os indivíduos interajam e relacionem-se, dessa forma, gerando sinergia.

Em processos de comunicação, os coletivos inteligentes materializam-se com a circulação de ideias, informações e conhecimentos. Porém, se visto desta forma simplificada, o seu entendimento ganha pouca proporção. Noutra perspectiva, os coletivos inteligentes podem ser percebidos como locais de simples troca de dados entre pessoas conhecidas ou, até mesmo, desconhecidas, via redes, comunidades ou internet. Nessa linha de pensamento, Costa (2008) propõe, então, um aprofundamento da discussão, observando a importância da interdependência entre as pessoas e o fim das fronteiras setoriais, como no caso das comunidades de prática, que podem limitar sua atuação aos grupos como também ampliar-se para a organização.

Logo, os coletivos inteligentes comprometem-se com a criação de formas de tratamento da informação (Berners-Lee, 2000; Rheingold, 1996) que devem ser caracterizadas pela sua distribuição e coordenação por todas as partes (Berners-Lee, 2000; Costa, 2005, 2008; Teixeira, 2005), gerando uma integração natural de todas as atividades humanas em todos os contextos.

A condição primária para os coletivos inteligentes e para a inteligência coletiva é o compartilhamento de informação e o conhecimento entre todos (Lévy, 1994). De cada indivíduo deve emergir o sentimento de interdependência e compartilhamento, visto vez que suas ações diárias influenciam e são influenciadas por outros indivíduos (Berners-Lee, 2000; Teixeira, 2005). Como resultados desse compartilhamento coletivo, são geradas duas possibilidades: o surgimento de meios mais simples e práticos de compreensão do trabalho coletivo; um melhor manejo dos instrumentos tecnológicos criados para a colaboração coletiva. Assim, um coletivo inteligente é uma percepção de interação a partir de ações coletivas que surgem, em um primeiro momento, no indivíduo e espalhamse pela sua rede de relações.

Por fim, frisa-se que, em um coletivo inteligente, nada é fixo. Isso não significa falta de ordem ou coordenação, mas sim que os atos são avaliados e gerenciados em tempo real, a partir de uma grande quantidade de critérios e também nos contextos mais diversos possíveis. Os relacionamentos não se limitam apenas a uma comunidade porque as pessoas incorporam características de multiplicidade e de 
troca. A metamorfose é a única característica permanente na inteligência coletiva e nos coletivos inteligentes (Costa, 2008; Teixeira, 2005).

\section{A tecnologia da informação orientada para grupos}

Uma característica marcante do uso da tecnologia da informação pelas organizações é a sua capacidade de possibilitar e condicionar ambientes para a realização do trabalho coletivo, o que permite aos grupos uma comunicação mais apropriada, a efetivação de tarefas compartilhadas, o gerenciamento das informações e a troca mútua de conhecimentos (Candotti \& Hoppen, 1999; Woodward, 2011). Além disso, a tecnologia da informação pode dar o suporte adequado para as redes organizacionais e para os processos de tomada de decisão (Ribeiro, 2005). Dessa maneira, por conta do uso da TI, são amplificadas, nas organizações, as possibilidades do trabalho em equipe (Christopoulos, 2008; Correia, Paulos, \& Mesquita, 2010).

Nesse contexto da TI, as redes podem ser percebidas como conjuntos "de transações repetidas e sustentadas por configurações relacionadas e estruturadas dotadas de fronteiras dinâmicas e elementos interconectados" (Balestrin, Verschoore, \& Reyes, 2010, p. 463). Essas configurações relacionadas potencializam a comunicação organizacional e as ações coletivas, como colaboração, cooperação e compartilhamento. Por conta disso, as redes logo se tornam particularmente importantes para esta investigação, por servirem de base para as comunidades de prática.

Wenger, White e Smith (2009), ao discutirem o uso da tecnologia da informação em comunidades de prática, ressaltam que as ferramentas são cada vez mais desenvolvidas numa perspectiva comunitária e que o surgimento de novas aplicações é tão rápido que as próprias comunidades não conseguem acompanhar.

Numa perspectiva histórica, denota-se a emergência do campo de pesquisa interdisciplinar que estuda as formas de trabalho em grupo e as tecnologias da informação que auxiliam tais processos, chamados de Computer Supported CooperativeWork (CSCW) (Coleman \& Khanna, 1995; Herrmann, 2009), os quais propõem melhores formas para suportar a atuação dos grupos, observando as distâncias geográficas, as formas de comunicação e o trabalho cooperativo (Xueguang, 2004). Como resultado, há tecnologias da informação voltadas para colaboração, cooperação e compartilhamento do trabalho em grupo, mais conhecidas como groupware. Exemplos de tecnologias utilizadas para cada função groupware estão expostos na Tabela 2.

Tabela 2

\section{As Três Funções Groupware e as Tecnologias Nelas Utilizadas}

\begin{tabular}{ll}
\hline Função groupware & Tecnologias utilizadas \\
\hline Comunicação & Chat, e-mail e videoconferência \\
Coordenação & Agenda eletrônica e workflow \\
Colaboração & $\begin{array}{l}\text { Sistemas de apoio à decisão em grupos (SAD-G), sistemas de reunião e conferência } \\
\text { eletrônica }\end{array}$ \\
\hline
\end{tabular}

Nota. Fonte: Baseado em Candotti, C. T., \& Hoppen, N. (1999, setembro). Reunião virtual e o uso de groupware: uma nova possibilidade de realizar trabalho em grupo. Anais do Encontro Nacional da Associação Nacional de Pós-Graduação e Pesquisa em Administração, Foz do Iguaçu, PR, Brasil, 23; Turban, E., Aronson, J. E., \& Liang, T. P. (2005). Decision support systems and intelligent systems. Tualatin, OR, USA: Prentice Hall.

Moeckel e Forcellini (2007) relatam que, entre os recursos de groupware existentes, destacamse, nos contextos coletivos, os fóruns e as listas de discussão eletrônicas, já que lhes são atribuídas funções de disseminar informações, promover as trocas de experiências e registrar lições aprendidas. 
Por fim, relata-se que, em coletivos virtuais, esses recursos conseguem promover o mais elevado nível de interação entre os participantes, por oferecerem espaços para opiniões e discussões entre membros (Primo, 2003).

\section{Metodologia}

Ao relembrar que o contexto deste artigo envolve a análise de elementos organizacionais voltados para ações de colaboração e cooperação baseadas em tecnologia da informação, percebe-se que este é um fenômeno em franca transformação e que apresenta, em suas conjunturas, diversas facetas as quais, até o momento, foram pouco estudadas. A partir dessa percepção, este relato assume uma metodologia exploratória e descritiva com o uso de métodos qualitativos, todos alinhados aos enquadramentos ontológicos e epistemológicos da perspectiva positivista (Morgan \& Smircich, 1980).

Ao considerar a harmonia do objeto estudado com as abordagens e métodos eleitos, decidiu-se por selecionar o estudo de caso único, com unidades de análise, como estratégia ideal para a investigação (Yin, 2010). Em consequência, o Conselho Nacional das Entidades de Provedores de Serviços de Internet (CONAPSI) foi escolhido como o caso a se estudar nesta pesquisa, e as unidades foram as oito associações que o compõem e que são listadas na Tabela 3.

Tabela 3

\section{Associações Membros do CONAPSI}

\begin{tabular}{ll}
\multicolumn{1}{c}{ SIGLA } & \multicolumn{1}{c}{ ASSOCIAÇÃO } \\
\hline ABRAMULTI & Associação Brasileira de Provedores de Internet \\
ABRANET & Associação Brasileira de Internet \\
ABRAPPIT & Associação Brasileira de Pequenos Provedores de Internet e Telecomunicações \\
ABRINT & Associação Brasileira de Provedores de Internet e Telecomunicações \\
APROVA-PE & Associação dos Provedores de Valor Adicionado e Comunicação Multimídia - PE \\
INTERNETSUL & Associação dos Provedores de Serviços e Informações da Internet \\
RGI & Rede Global Info \\
REDETELESUL & Associação Nacional das Empresas de Soluções de Internet e Telecomunicações \\
\hline
\end{tabular}

Nota. Fonte: Dados da pesquisa.

Foram dois os motivos que circunstanciaram a escolha do CONAPSI como caso da pesquisa. Em princípio, há o fato de que esse conselho reúne as principais e mais importantes associações de provedores de internet do país, agregando mais de duas mil empresas que atuam no ramo. Dados da ANATEL (2011) apontam para a existência de 2.425 empresas de internet banda larga no Brasil e demonstram que o CONAPSI consegue aglutinar mais de $80 \%$ dos provedores do país, sendo, por isso, muito representativo.

A configuração organizacional do CONAPSI surgiu como segundo elemento motivador, por congregar, em seu interior, características comuns a outros casos que se consideram como a realidade das comunidades de prática, tais como rol de membros e área de atuação. Isso eleva o CONAPSI ao posto de representante das associações, quiçá $\mathrm{CoP}$, permitindo que os resultados desta investigação possam ser considerados em casos semelhantes de organizações que também se estruturam como CoP. 
Na sequência, é apresentado na Tabela 4 o desenho da pesquisa com a finalidade de ilustrar, de forma serial e lógica, as fases operacionais do estudo que, neste caso, foi planejado para acontecer em duas etapas.

Tabela 4

Desenho da Pesquisa

\begin{tabular}{|c|c|c|c|c|c|}
\hline \multicolumn{2}{|c|}{ ETAPA/MÉTODO } & \multirow{2}{*}{$\begin{array}{l}\text { META } \\
\text { Contextualizar o } \\
\text { cenário e identificar } \\
\text { os três aspectos das } \\
\text { CoP (domínio, } \\
\text { comunidade e } \\
\text { prática). }\end{array}$} & \multirow{2}{*}{$\begin{array}{l}\text { BASE TEÓRICA } \\
\text { Wenger (2001), } \\
\text { Easterby-Smith, } \\
\text { Crossan e Nicolini } \\
\text { (2000), Souza-Silva } \\
(2009)\end{array}$} & \multirow{2}{*}{$\begin{array}{l}\text { DADOS } \\
\text { Listas de discussão } \\
\text { eletrônicas do } \\
\text { CONAPSI, com } \\
\text { duração de três } \\
\text { meses }\end{array}$} & \multirow{2}{*}{$\begin{array}{l}\text { TÉCNICA DE } \\
\text { TRATAMENTO } \\
\text { DOS DADOS } \\
\begin{array}{l}\text { Estatística } \\
\text { descritiva }\end{array}\end{array}$} \\
\hline 1 & $\begin{array}{l}\text { Levantamento do } \\
\text { cenário e } \\
\text { enquadramento: } \\
\text { pesquisa } \\
\text { documental }\end{array}$ & & & & \\
\hline 2 & $\begin{array}{l}\text { Estudo de caso: } \\
\text { entrevistas } \\
\text { semiestruturadas }\end{array}$ & $\begin{array}{l}\text { Analisar as ações } \\
\text { coletivas suportadas } \\
\text { pela tecnologia da } \\
\text { informação - } \\
\text { colaboração, } \\
\text { cooperação e } \\
\text { compartilhamento. }\end{array}$ & $\begin{array}{l}\text { Argyle (1991), Boavida } \\
\text { e Ponte (2002), Goco } \\
(2006), \text { Costa }(2008)\end{array}$ & $\begin{array}{l}\text { Relatos dos oito } \\
\text { empresários } \\
\text { representantes das } \\
\text { associações }\end{array}$ & $\begin{array}{l}\text { Análise de } \\
\text { conteúdo (Bardin, } \\
\text { 2009) }\end{array}$ \\
\hline
\end{tabular}

Nota. Fonte: Planejamento da pesquisa.

Conforme ilustrado na Tabela 4, os dados foram coletados em dois momentos: no primeiro, utilizou-se pesquisa documental, e, no segundo, foram realizadas entrevistas semiestruturadas. O objetivo da pesquisa documental foi o de pôr os pesquisadores em contato inicial com o campo, para que pudessem identificar vestígios da existência dos três aspectos norteadores das comunidades de prática (domínio, comunidade e prática) nas situações estudadas. Mediante acesso às listas de discussão eletrônicas do CONAPSI, dos meses de março a maio de 2011 (totalizando 843 mensagens), buscou-se mapear quais eram os temas discutidos, como eram as formas de engajamento dos membros e quais as maneiras de interação e troca de conteúdos entre eles. Os registros produzidos neste primeiro momento da pesquisa auxiliaram no levantamento do cenário e no enquadramento do CONAPSI como uma CoP a estudar.

Já as oito entrevistas semiestruturadas empreendidas no segundo momento da investigação tiveram como público-alvo os empresários representantes legais das associações de provedores de internet que compõem o CONAPSI. O roteiro de entrevista foi construído a partir de um quadro de consistência, criado no planejamento da pesquisa, em que se buscou encontrar a aderência necessária entre o problema central, os objetivos e as questões formuladas. Também orientou a confecção deste roteiro um conjunto de sistemas de categorias de análises predefinidas, que será apresentado na Tabela 5 , adiante.

Os dados referentes à pesquisa documental foram tratados com estatística descritiva, o que possibilitou a elaboração de tabulações de frequências para a análise das características gerais das associações de provedores de internet que compõem o CONAPSI. Já os dados das entrevistas foram tratados com a técnica de análise de conteúdo (Bardin, 2009). As três fases que compuseram o procedimento foram: (a) pré-análise, na qual se verificou a seleção dos conteúdos avaliados; (b) análise, em que se empreendeu primeiramente a categorização dos conteúdos e a elaboração de resumos descritivos; e (c) interpretação, em que as sínteses dos conteúdos e inferências foram feitas. Reforça-se que o conjunto de sistemas de categorias de análise predefinido norteou as análises e as interpretações dos dados. Esses sistemas estão espelhados a seguir. 
Tabela 5

Conjunto de Sistemas de Categorias Predefinidas para a Pesquisa

\begin{tabular}{|c|c|c|}
\hline \multicolumn{3}{|c|}{ SISTEMA A: Colaboração - CLB } \\
\hline CATEGORIA & DESCRIÇÃO & INDICADORES \\
\hline $\begin{array}{l}\text { CLB } 1 \\
\text { Políticas }\end{array}$ & $\begin{array}{l}\text { Descreve as políticas que apoiam a } \\
\text { participação na comunidade de } \\
\text { prática. }\end{array}$ & $\begin{array}{l}\text { Formas de participação e } \\
\text { engajamentos }\end{array}$ \\
\hline $\begin{array}{l}\text { CLB } 2 \\
\text { Estratégias }\end{array}$ & $\begin{array}{l}\text { Descreve as estratégias que apoiam } \\
\text { as ações de interação na } \\
\text { comunidade de prática. }\end{array}$ & $\begin{array}{l}\text { Criação e manutenção de meios } \\
\text { para interação }\end{array}$ \\
\hline $\begin{array}{l}\text { CLB } 3 \\
\text { Incentivos à colaboração }\end{array}$ & $\begin{array}{l}\text { Descreve os incentivos que buscam } \\
\text { promover a colaboração entre os } \\
\text { membros da comunidade de prática. }\end{array}$ & $\begin{array}{l}\text { Natureza dos incentivos } \\
\text { Barreiras à colaboração }\end{array}$ \\
\hline \multicolumn{3}{|c|}{ SISTEMA B: Cooperação - CPR } \\
\hline CATEGORIA & DESCRIÇÃO & INDICADORES \\
\hline $\begin{array}{l}\text { CPR } 1 \\
\text { Processos }\end{array}$ & $\begin{array}{l}\text { Descreve os processos e as tarefas } \\
\text { voltados para a cooperação entre os } \\
\text { membros da comunidade de prática. }\end{array}$ & Natureza dos processos \\
\hline $\begin{array}{l}\text { CPR } 2 \\
\text { Hábitos de cooperar }\end{array}$ & $\begin{array}{l}\text { Descreve os hábitos dos membros } \\
\text { de cooperação na comunidade de } \\
\text { prática. }\end{array}$ & $\begin{array}{l}\text { Canais utilizados para cooperar } \\
\text { Estímulos à cooperação } \\
\text { Barreiras à cooperação }\end{array}$ \\
\hline \multicolumn{3}{|c|}{ SISTEMA C: Compartilhamento via tecnologia da informação - CTI } \\
\hline CATEGORIA & DESCRIÇÃO & INDICADORES \\
\hline $\begin{array}{l}\text { CTI } 1 \\
\text { Meios tecnológicos }\end{array}$ & $\begin{array}{l}\text { Descreve as tecnologias da } \\
\text { informação utilizadas na } \\
\text { comunidade de prática. }\end{array}$ & $\begin{array}{l}\text { Ferramentas utilizadas } \\
\text { Finalidades do uso }\end{array}$ \\
\hline $\begin{array}{l}\text { CTI } 2 \\
\text { Comunicação }\end{array}$ & $\begin{array}{l}\text { Descreve as funções da } \\
\text { comunicação na comunidade de } \\
\text { prática. }\end{array}$ & $\begin{array}{l}\text { Características } \\
\text { Finalidades }\end{array}$ \\
\hline $\begin{array}{l}\text { CTI } 3 \\
\text { Contribuições da tecnologia da } \\
\text { informação }\end{array}$ & $\begin{array}{l}\text { Descreve como a tecnologia da } \\
\text { informação estrutura e contribui } \\
\text { para o compartilhamento de } \\
\text { conteúdos na comunidade de } \\
\text { prática. }\end{array}$ & $\begin{array}{l}\text { Estruturantes } \\
\text { Para compartilhamento }\end{array}$ \\
\hline
\end{tabular}

Nota. Fonte: Planejamento da pesquisa.

Ainda, é necessário mencionar que as categorias predefinidas emergiram da literatura que embasou a investigação, sendo que cada sistema foi direcionado para uma ação específica na busca da resolução do objetivo geral do estudo.

\section{Resultados}

As análises aqui realizadas são fruto das duas etapas propostas para a pesquisa, sendo a primeira direcionada ao levantamento do cenário e ao enquadramento das associações do CONAPSI como uma comunidade de prática, a partir da pesquisa documental em listas de discussão eletrônicas; e a segunda, 
à observância dos relatos das entrevistas semiestruturadas, dos representantes das associações como unidades que compõem o estudo de caso. A Tabela 6 sintetiza o rol de dados coletados na investigação.

Tabela 6

Situação Geral dos Dados

\begin{tabular}{cccc}
\hline ETAPA & MÉTODO & QTD. DADOS & PERÍODO COLETA \\
\hline $\begin{array}{c}\text { Levantamento do cenário } \\
\text { e enquadramento das } \\
\text { associações }\end{array}$ & Pesquisa documental & 843 mensagens & Março a maio de 2011 \\
$\begin{array}{c}\text { Estudo de caso da CoP } \\
\text { CONAPSI }\end{array}$ & Entrevista & 8 entrevistas & Junho a outubro de 2011 \\
\hline
\end{tabular}

Nota. Fonte: Dados da pesquisa.

\section{Levantamento do cenário e enquadramento das associações}

Esta etapa da investigação teve o propósito de contextualizar o cenário em que as associações integrantes do Conselho Nacional das Entidades de Provedores de Serviços de Internet (CONAPSI) estão inseridas. A pesquisa documental empreendida aqui buscou identificar na organização os três aspectos marcantes das CoP descritos na literatura (Wenger, 2001), que são o domínio, a comunidade e a prática. O conteúdo da Tabela 7 foi extraído das particularidades encontradas no cotidiano das discussões travadas nas listas eletrônicas e demonstra, sinteticamente, as características da atuação dos membros do CONAPSI.

Tabela 7

Os Aspectos e as Características das Comunidades de Prática Investigados no Levantamento do Cenário e no Enquadramento das Associações

\begin{tabular}{ll}
\hline ASPECTOS & CARACTERÍSTICAS \\
\hline Domínio & $\begin{array}{l}\text { Origens dos temas (técnicos, empresariais, regulatórios, políticos). } \\
\text { Importância dos temas. }\end{array}$ \\
$\begin{array}{l}\text { Comunidade } \\
\text { Prática }\end{array}$ & $\begin{array}{l}\text { Ouantidade e tipos de participação de membros (principal, periférica, transacional, passiva). } \\
\text { experiências, compartilhar informações, reivindicar, realizar negócios). }\end{array}$ \\
\hline
\end{tabular}

Nota. Fonte: Dados da pesquisa.

Os principais tópicos de discussão que definem o domínio e o campo de atuação do CONAPSI, bem como das associações que o integram, estão voltados para os temas técnicos e empresariais. Entre o rol de tópicos técnicos, foram listadas discussões sobre o uso de sistemas e equipamentos, especificações e configurações de produtos, entre outros. Por sua vez, entre os temas empresariais, foram identificadas negociações, discussões sobre oportunidades para compras coletivas de materiais e gestão dos negócios. Outras características do aspecto domínio foram:

- Tópicos de discussão: nas listas de discussão eletrônicas, os tópicos de discussão são definidos pelos próprios participantes, a partir das suas necessidades. Já nos encontros, os tópicos são estruturados e definidos pelos organizadores.

- Mecanismos de gestão: não há, por parte da gestão do CONAPSI, incentivos formais para a participação ou diretrizes sobre a gestão das listas eletrônicas, ficando a cargo dos próprios 
participantes a administração dos conteúdos, numa espécie de modelo aberto de interação e autogestão.

- Modalidades de intervenção: nos eventos, independentemente do tema em pauta, é comum que surjam participações com a finalidade de dirimirem dúvidas. Já para compartilhar experiências ou informações, só foram percebidas intervenções durante as explanações técnicas.

- Modalidades de participação: enquanto nas listas de discussão eletrônicas as participações dos membros configuram-se como ativas, nos eventos elas caracterizam-se como amenas, fortalecendo $\mathrm{o}$ interesse pela participação virtual.

As características listadas levam a crer que, de modo geral, o CONAPSI detém temas de discussão e áreas de atuação claros, fazendo com que seus integrantes tenham o entendimento de que estão inseridos num contexto que apresenta contornos organizacionais bem-definidos, embora não institucionalizados e pouco estruturados, a partir do seu domínio. Tais constatações corroboram o entendimento de diversos autores (Schommer, 2005; Wenger et al., 2002), quando estes afirmam que um dos pilares de sustentação das CoP é, exatamente, a clareza dos conteúdos discutidos nos grupos.

Já o aspecto comunidade se fez presente no CONAPSI, uma vez que foi averiguado que existem dois grupos de membros ativos, que são: o principal, que é fortemente comprometido com a organização (mesmo que informalmente) e é responsável pela maioria dos temas postos em pauta; e o de participação periférica, que demonstra um comprometimento menor com o coletivo. Aqueles que formam o grupo principal correspondem a 12,5\% dos membros ativos, participando, em médias mensais, mais de seis vezes das listas de discussões. A seu turno, os integrantes do grupo de participação periférica somam 87,5\% do total de membros, computando uma média de duas a cinco participações mensais.

Outra característica marcante relacionada ao aspecto comunidade é que as participações virtuais, por intermédio das listas de discussão, estão mais consolidadas no CONAPSI do que as participações presenciais, que acontecem durante os eventos promovidos. É provável que os membros optem pela institucionalização da virtualidade, já que ela propicia facilidades de comunicação, minimiza barreiras geográficas e temporais e reduz custos. Interessante notar que Wenger et al. (2009), já naquela época, apontavam para o fato de que a institucionalização da virtualidade parecia ser um processo natural em comunidades de prática.

Por seu turno, a observação do aspecto prática revelou que os membros do CONAPSI percebem as listas de discussão eletrônicas como bases coletivas de consulta, já que elas são utilizadas como espaços de compartilhamento de conteúdos e debates variados. Ainda foi percebido que as participações nos coletivos virtuais acontecem espontaneamente, principalmente quando surgem temas técnicos. Nessas situações, os conteúdos são cambiados livremente, porém esse fato não se repete quando temas empresariais estão em pauta.

As análises dos conteúdos das listas de discussão eletrônicas ainda revelaram a existência de espirais de construção coletiva de conhecimento, que surgem quando membros aglutinados desenvolvem novos conhecimentos a partir das trocas de dados, informações e experiências dentro dos contextos virtuais. Acredita-se que isso ocorra pelo fato de que as listas, pela sua própria natureza, acabam por estimular as participações e interações, gerando uma sensação de confiança coletiva.

Por fim, relata-se que todas as características listadas nesta seção trazem o entendimento de que o CONAPSI, incluindo as oito associações que o compõem, caracteriza-se efetivamente como uma comunidade de prática no cenário da pesquisa, e que produz inteligência coletiva, visto que os três aspectos centrais foram evidenciados pelos dados colhidos. Em reforço, os seus membros veem esta CoP como uma organização que possui domínios definidos e que passa por um processo de institucionalização dos espaços virtuais, com uso efetivo das listas de discussão eletrônicas. Ao estimularem as participações dos membros como um processo natural, essas listas proporcionam o surgimento de espirais de construção coletiva de conhecimento, configurando contornos de coletivo inteligente. 
A Figura 2, a seguir, tem a função de ilustrar, de forma esquematizada, a existência dos três aspectos (domínio, comunidade e prática), a partir das características detectadas em campo, chegando à comunidade de prática CONAPSI propriamente constituída.

\section{CARACTERÍSTICAS}

ASPECTOS

COMUNIDADE

DE PRÁTICA

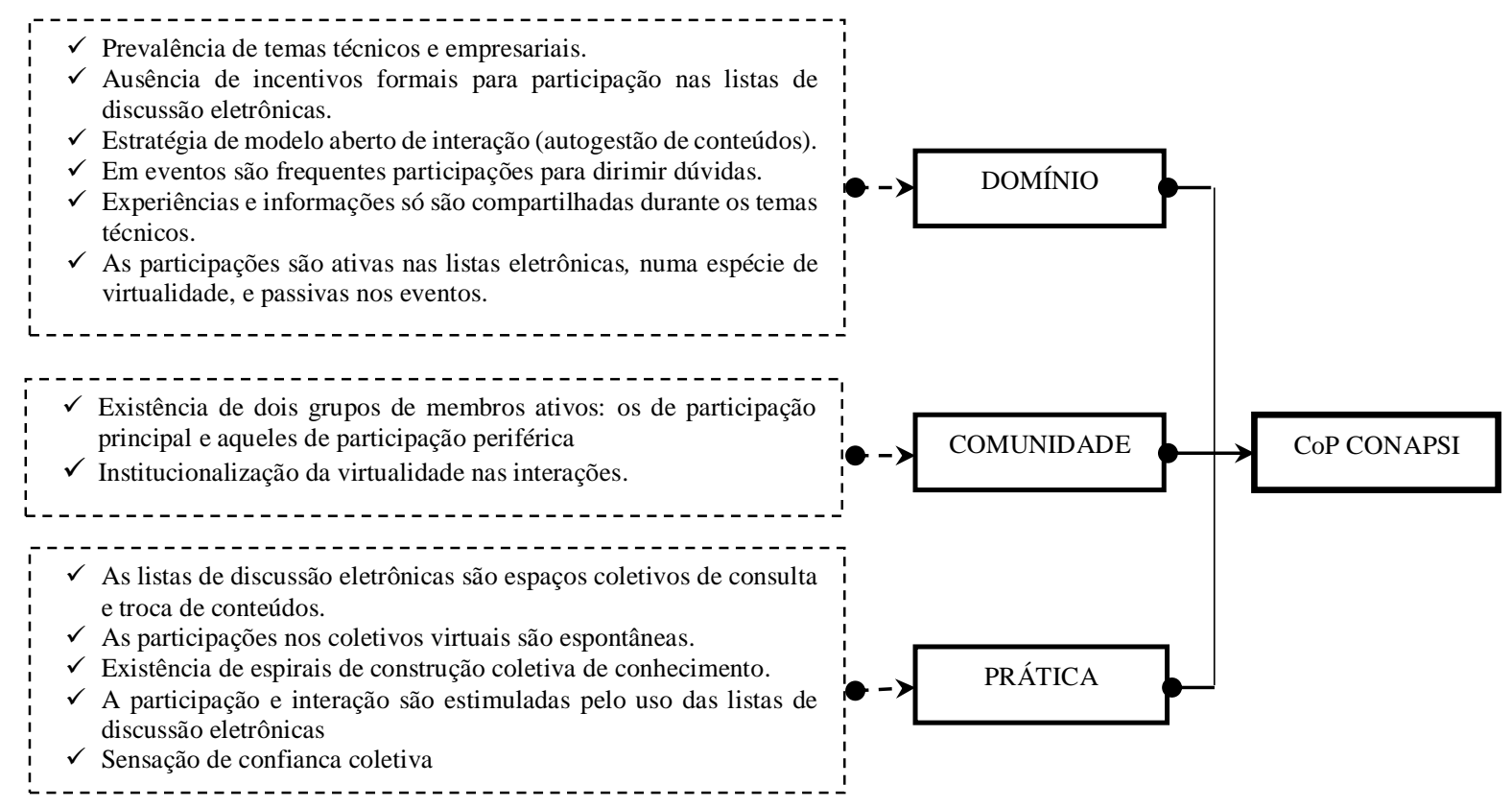

Figura 2. Características e Aspectos que Compõem a CoP CONAPSI.

Fonte: Dados da pesquisa.

\section{Estudo de caso da CoP CONAPSI}

Esta seção destina-se a examinar os resultados das entrevistas realizadas com os representantes das oito associações que compõem o CONAPSI. É importante relembrar que se utilizou como estratégia, nesta etapa da investigação, o estudo de caso único com diversas unidades, sendo o CONAPSI considerado o caso e as associações integrantes suas unidades de análise.

Como já apresentado na Tabela 5, foram três os sistemas de categorias que estruturam as análises realizadas nesta etapa: (a) colaboração, em que foram investigadas as políticas, as estratégias e os incentivos à colaboração; (b) cooperação, os processos e os hábitos de cooperar; e (c) compartilhamento via tecnologia da informação, que evidenciou os meios tecnológicos, a comunicação e as contribuições da tecnologia da informação.

\section{Colaboração}

O CONAPSI visa, por definição, promover formas de aproximação dos seus membros, com vistas a incentivar as interações e as trocas de conteúdos. Para esse fim, utilizam-se como estratégias principais as listas de discussão eletrônicas e os eventos presenciais promovidos ao logo do ano.

Foi facilmente percebido que os associados creditam às listas eletrônicas as reais possibilidades de colaboração, pois, por serem permanentes, mostram-se como repositórios de conteúdos satisfatórios e canais de fácil acesso.

Em razão de não existirem políticas formais de incentivo à interação, o CONAPSI limita-se a fornecer o suporte técnico necessário para o funcionamento das listas eletrônicas de discussão, não moderando os conteúdos destas, que ficam a critério dos próprios associados. Como consequência, há o 
surgimento espontâneo de regras coletivas de auxílio, que se destinam a ajudar os membros necessitados, tirando dúvidas ou cedendo informações pelas listas. Os relatos a seguir fortalecem tal entendimento:

"As políticas se formaram espontaneamente. Por ser uma atividade nova, provedor de acesso à Internet, todos buscam se ajudar através de troca de e-mails, através de uma lista cadastrada." (ASSOCIADO B)

"O papel do CONAPSI é o de promover a aproximação entre os seus associados para a partilha e troca de informações." (ASSOCIADO F)

Ainda quanto ao exposto, vale mencionar que Wenger et al. (n.d.), ao relacionarem as tecnologias da informação utilizadas por grupos, relatam que as listas de discussão eletrônicas são ferramentas comumente encontradas nas CoP, visto que lhes são atribuídas a função de serem canais de disseminação de conteúdos nos coletivos.

Os associados ouvidos ratificaram a percepção sobre taxar-se como principais estratégias à colaboração a realização de eventos e a criação das listas de discussão eletrônicas. Com menor intensidade de reconhecimento e como estratégia colaborativa, também, surgiu na pesquisa a ação de compras coletivas, que se revelara como uma iniciativa advinda dos próprios associados, sem que o Conselho atuasse de maneira ativa no processo.

Complementando, apareceram na categoria incentivos à colaboração aqueles estímulos de natureza financeira, cooperativa e de acesso ao conhecimento via listas de discussão eletrônicas. Os incentivos financeiros surgiram com as possibilidades de ganhos econômicos a partir das compras coletivas de produtos e serviços. Já os incentivos de natureza cooperativista voltaram-se à conscientização do valor coletivo e ao fortalecimento do conselho como entidade representativa; por fim, a possibilidade de acesso ao conhecimento, por si só, mostrou-se como um tipo de incentivo à colaboração, fazendo com que os provedores se filiassem ao CONAPSI, a fim de que pudessem ter acesso aos conteúdos das listas de discussão.

Assim, em resumo, a colaboração na CoP CONAPSI caracterizou-se pela ausência de políticas organizacionais formais de incentivo à colaboração que, na prática, foram substituídas pela espontaneidade das regras coletivas de auxílio. As estratégias de interação da CoP são depositadas nas listas de discussão eletrônicas, as quais são utilizadas como canais que possibilitam acesso aos conhecimentos compartilhados pelos demais membros, e esse acesso é um forte incentivador à participação e à colaboração.

A realidade aqui exposta torna viável afirmar que o coletivo inteligente surge na comunidade de prática do CONAPSI de forma livre, não dependendo dos direcionamentos formais da organização, já que um de seus principais elementos configuradores reside no próprio desejo dos membros de colaborarem entre si, como constatado na análise desta categoria.

\section{Cooperação}

A virtualidade nos processos é elemento presente nos dois tipos de cooperação identificados na pesquisa, sendo que neles existem variações de efetividade no uso da TI. Nos processos de natureza voltados para o engajamento (fóruns de discussão e grupos de trabalho com temas específicos), são utilizadas ferramentas básicas de tecnologia da informação, como e-mails pessoais para comunicação entre pares. Foi identificado, também, que a tecnologia da informação ganha uso em fóruns de discussão on-line que acontecem de maneira esporádica.

Já nos processos voltados para os negócios, as listas de discussão eletrônicas são bastante utilizadas como suportes tecnológicos, em especial para as redes que surgem a partir dos relacionamentos que objetivam realização de compras coletivas.

Para os entrevistados, a cooperação na CoP CONAPSI também acontece através das trocas de conteúdos nas listas de discussão que têm a virtualidade como ambiente central. Em complemento, os dados levam a crer que o principal elemento motivador para a participação dos membros na CoP é a 
cooperação de conteúdos que acontece no ambiente virtual das listas de discussão eletrônicas, e os relatos adiante comprovam isso:

"Dispomos de uma lista de discussão que é hoje a principal ferramenta e meio utilizado para o compartilhamento de informações. Neste meio, as informações são postadas tanto pela diretoria da entidade como por qualquer associado." (ASSOCIADO G)

"A maioria [dos associados] procura compartilhar os seus conhecimentos. Existe o incentivo nas listas [eletrônicas], muitas vezes, através de conversas privadas e também via TI. O pessoal vê as listas como redes de relacionamentos.” (ASSOCIADO A)

Os estímulos à cooperação que surgem na CoP CONAPSI estão alinhados com o uso das listas de discussão eletrônicas (canais virtuais), já que estão voltados para as trocas de conteúdos. Em adição, a efetiva cooperação tropeça em barreiras ligadas à participação dos membros, que, por vezes, configurase de maneira apática, passiva e pouco motivada.

Em síntese, existe uma forma de cooperação na CoP CONAPSI que se caracteriza como sendo virtualizada e tem como objeto central a troca de conteúdos, em particular, de informações, resultados de experiências e conhecimentos acumulados. Goco (2006) lembra que a cooperação, ao estimular as relações interindividuais, acaba por criar sistemas de interação, como acontece com os membros da CoP estudada.

As interações oportunizadas pelos espaços virtuais geram redes de relacionamentos, com traços de coletivo inteligente, e recebem o aporte da TI em seus elos. Os maiores empecilhos à cooperação estão nas participações apáticas dos membros.

Neste cenário, pode-se afirmar que, sem o ambiente virtual proporcionado pelas listas de discussão eletrônicas, a CoP CONAPSI não teria ações de cooperação efetivas, nem, consequentemente, configurações de um coletivo inteligente.

\section{Compartilhamento via tecnologia da informação}

Os dados analisados neste sistema de categorias comprovaram o que já tinha sido notado nos outros sistemas: as listas de discussão eletrônicas são os principais meios tecnológicos de groupware (tecnologia colaborativa) utilizados pelos membros da CoP CONAPSI. Entre as diversas funções que lhes são atribuídas, estão a comunicação e a redução das barreiras geográficas.

"Sem elas [as listas de discussão eletrônicas] seria inviável a troca de informações entre os associados. O uso dessas tecnologias propiciou novas possibilidades de geração colaborativa e cooperativa de conhecimento." (ASSOCIADO H)

Sobre a comunicação, esta se caracteriza como fortemente suportada pela tecnologia da informação e tem a função central de promover a mobilização em torno de temas importantes, assim, atuando como canal de interação entre os associados e a entidade.

O uso efetivo das listas eletrônicas provoca uma redução da assimetria do conhecimento entre os associados, pois foi comum identificar provedores mais experientes repassando conteúdos para aqueles neófitos. Outro aspecto interessante anotado a partir do campo foi o surgimento natural dos repositórios virtuais de pesquisa, uma vez que os históricos das mensagens ficam armazenados e podem ser consultados a qualquer tempo. Em complemento, os relacionamentos entre os membros também recebem contribuições, pois se abrem possibilidades, transparência e aglutinação em torno de temas relevantes.

Pode-se resumir colocando que o compartilhamento via tecnologia da informação na CoP CONAPSI, assim como acontece com a colaboração e a cooperação, caracteriza-se pelo uso efetivo das listas de discussão eletrônicas, às quais foram atribuídas a função de canal de comunicação, auxílio na redução da assimetria do conhecimento e superação de barreiras geográficas. As contribuições da TI, 
além de proporcionarem o surgimento dos repositórios virtuais de pesquisa, estruturam os relacionamentos entre os membros, dando contornos da existência de um coletivo inteligente.

Relembrando Wenger et al. (2009, n.d.), pode-se notar que, na CoP CONAPSI, vários elementos sofrem influência direta da TI, entre eles: tempo e espaço, já que as limitações geográficas e temporais são superadas; conexões, pois as velocidades nas respostas melhoram a comunicação; adesão à comunidade, via aglutinação de membros; desenvolvimento comunitário, por existir transparência nas relações.

Para consolidar e complementar a exposição feita nesta seção, apresenta-se, a seguir, a Figura 3, que foi construída a partir dos elementos detectados na investigação e que representa a configuração do coletivo inteligente existente na CoP CONAPSI.

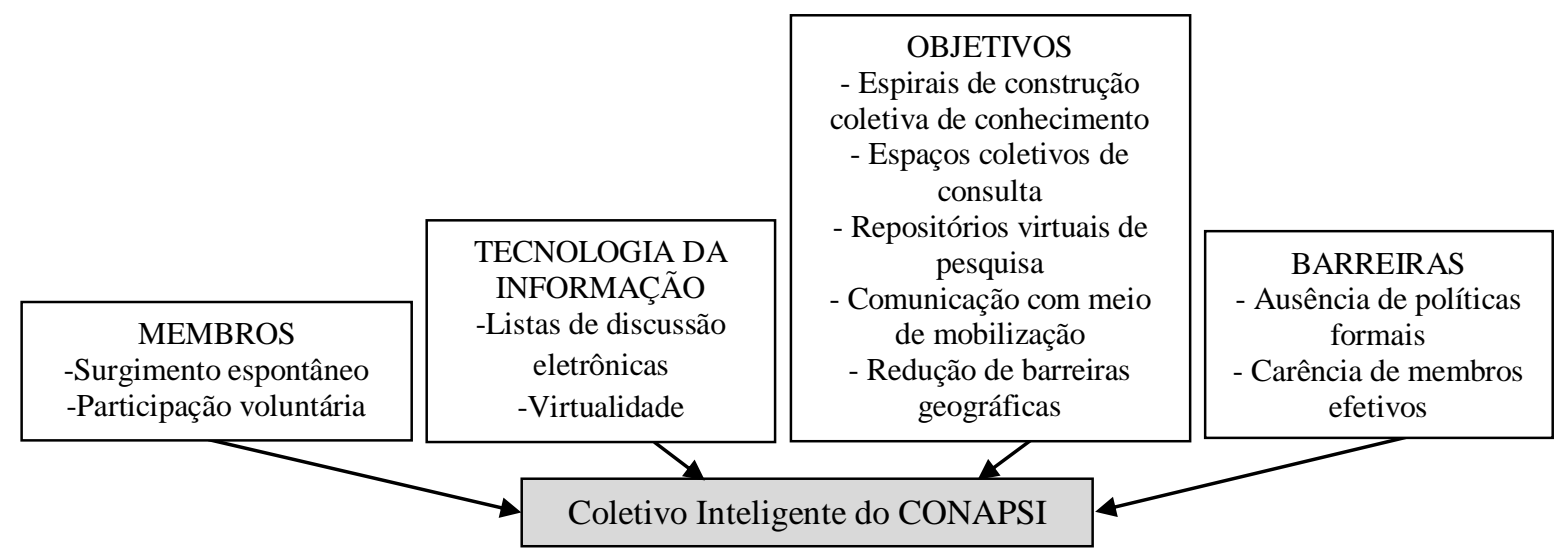

Figura 3. Configuração do Coletivo Inteligente da CoP CONAPSI.

Fonte: Dados da pesquisa.

A última seção deste trabalho, que vem na sequência, encarrega-se de apresentar conclusões da investigação, travestidas de considerações finais, como uma síntese do texto, dessa forma, buscando confrontar os objetivos propostos e os dados coletados em campo.

\section{Considerações Finais}

As duas etapas da investigação tiveram o objetivo central de analisar como as ações coletivas suportadas pela tecnologia da informação na comunidade de prática do CONAPSI caracterizam-se como elementos indutores à condição de que brotasse do mesmo um coletivo inteligente. As interpretações dos dados coletados na CoP CONAPSI levam a crer que as ações de colaboração e cooperação, bem como os compartilhamentos resultantes da comunicação, ao receberem aportes da tecnologia da informação, em particular das listas de discussão eletrônicas, elevam a comunidade de prática à condição de coletivo inteligente. Neste coletivo inteligente, a TI tem a função de estruturar iniciativas de interação e trocas de conteúdos entre os membros.

A produção de inteligência coletiva, em verdade concretizada pelas espirais de construção coletiva de conhecimento, também se mostrou fator determinante para o surgimento, configuração e manutenção do coletivo inteligente da CoP CONAPSI, bem como se revelou um resultado marcante nesta investigação.

Ao mencionar as limitações desta pesquisa, aponta-se como primeiro elemento o fato de apenas os conteúdos de três listas de discussão eletrônicas da associação estudada terem sido considerados, apesar das volumosas 843 mensagens. A generalização dos resultados de estudos de caso é vista como segunda, e quiçá mais relevante, limitação desta investigação, pois os dados são originários de apenas oito unidades de um único caso, o CONAPSI, e englobam um segmento particular de atuação, que é o 
dos provedores de internet banda larga. Por conta disso, é fato que as conclusões da pesquisa possuem limitada generalização e podem ser expandidas para outras realidades, desde que sejam consideradas as particularidades do estudo.

Os resultados obtidos a partir das investigações aqui realizadas levam a crer que o tema em questão, o uso da TI em comunidades de prática, merece que se realizem novas incursões, visto que são múltiplas as facetas a serem exploradas.

Nesse sentido, direcionamentos para futuras pesquisas são sugeridos aqui: estudar o uso da TI em comunidades de prática em outros segmentos; investigar as ações coletivas em outros arranjos organizacionais diferentes das comunidades de prática; realizar estudos comparativos entre as características das ações coletivas em espaços virtuais e não virtuais, incluindo as comunidades virtuais de práticas (CoVP); e investigar mais a fundo os fenômenos das espirais de construção coletiva de conhecimento.

De modo geral, espera-se que os resultados desta pesquisa contribuam também para maiores compreensão e aprofundamento do que sejam ações coletivas, coletivos inteligentes e consequências resultantes dos espaços virtuais nas organizações.

Estipula-se à atividade de pesquisa um script que a aproxime dos seres vivos e animados, em especial as pessoas inseridas nos complexos contextos sociais como as organizações. Conjugar essas animações em um ambiente coletivo, inteligente e também evolutivo é tarefa da academia e deve ser subordinada às ações de uso social da tecnologia da informação. A temática de CoP é descortinadora justa porque traz à tona esse gregarismo por vezes esquecido, mas reforçado pelo uso social da tecnologia para revigorar aquilo que, desde eras remotas, direciona a espécie humana à sabedoria, ao uso e à partilha de conhecimentos: sobrepujar as dificuldades. Trabalhar $\mathrm{CoP}$ em organizações com a TI é, como visto, um retorno, uma volta à origem.

Com essa visão, espera-se que o presente estudo provoque outros pesquisadores a debruçarem-se sobre as questões que envolvam esses coletivos inteligentes, tão facilmente produzíveis no mundo contemporâneo das organizações e da sociedade.

\section{Referências}

Agencia Nacional de Telecomunicações. (2011). Sistema de serviços de telecomunicações. Recuperado de http://sistemas.anatel.gov.br/stel/consultas/ListaPrestadorasServico/tela.asp?pNumServico $=045$

Araujo, L. (1998). Knowing and learning as networking. Management Learning, 29(3), 317-336. doi: 10.1177/1350507698293004

Argote, L. (2005). Reflections on two views of managing learning and knowledge in organizations. Journal of Management Inquiry, 14(1), 43-48. doi: 10.1177/1056492604273179

Argyle, M. (1991). Cooperation: the basis of sociability. London: Routledge.

Balestrin, A., Verschoore, J. R., \& Reyes, E., Jr. (2010). O campo de estudo sobre redes de cooperação interorganizacionais no Brasil. Revista de Administração Contemporânea, 14(3), 458-477. Recuperado de http://www.scielo.br/pdf/rac/v14n3/v14n3a05.pdf. doi: 10.1590/S141565552010000300005

Bardin, L. (2009). Análise de conteúdo. Lisboa: Edições 70.

Berners-Lee, T. (2000). Tejiendo la red: el inventor del world wide web nos descubre su origem. Madri: Siglo XXI. 
Boavida, A. M., \& Ponte, J. P. (2002). Investigação colaborativa: potencialidades e problemas. In Grupo de Trabalho sobre Investigação (Org.), Refletir e investigar sobre a prática profissional (pp. 4355). Lisboa: APM.

Brandon, T., \& Charlton, J. (2011). The lessons learned from developing an inclusive learning and teaching community of practice. International Journal of Inclusive Education, 15(1), 165-178. doi: 10.1080/13603116.2010.496214

Candotti, C. T., \& Hoppen, N. (1999, setembro). Reunião virtual e o uso de groupware: uma nova possibilidade de realizar trabalho em grupo. Anais do Encontro Nacional da Associação Nacional de Pós-Graduação e Pesquisa em Administração, Foz do Iguaçu, PR, Brasil, 23.

Christopoulos, T. (2008). A sustentação das comunidades virtuais de aprendizagem e de prática (Tese de doutorado). Universidade de São Paulo, São Paulo, SP, Brasil.

Coleman, D., \& Khanna, R. (1995). Groupware technology and applications: an overview of groupware. New Jersey: Prentice Hall.

Correia, A. M. R., Paulos, A., \& Mesquita, A. (2010). Virtual communities of practice: investigating motivations and constraints in the processes of knowledge creation and transfer. Electronic Journal of Knowledge Management, 8(1), 11-20.

Costa, R. da (2005). Por um novo conceito de comunidade: redes sociais, comunidades pessoais, inteligência coletiva. Interface - Comunicação, Saúde, Educação, 9(17), 235-248. doi: 10.1590/S1414-32832005000200003

Costa, R. da (2008). Inteligência coletiva: comunicação, capitalismo cognitivo e micropolítica. Famecos, 1(37), 61-68.

Dubrin, A. J. (2003). Fundamentos do comportamento organizacional. São Paulo: Thomson.

Easterby-Smith, M., Crossan, M., \& Nicolini, D. (2000). Organizational learning debates: past, present and future. Journal of Management Studies, 37(6), 783-796. doi: 10.1111/1467-6486.00203

Gazzoli, P. (2012). Comunidades de prática enquanto viabilizadoras de projetos comuns em ambientes turbulentos: uma abordagem crítica. Revista de Administração Contemporânea, 16(6), 806-826. Recuperado de http://www.scielo.br/pdf/rac/v16n6/a04v16n6.pdf. doi: 10.1590/S141565552012000600004

Gherardi, S., \& Nicolini, D. (2000). The organizational learning of safety in communities of practices. Journal of Management Inquiry, 9(1), 7-18. doi: 10.1177/105649260091002

Goco, A. L. P. (2006). Cooperação versus colaboração: conceitos para o ensino de enfermagem em ambiente virtual. Revista Brasileira de Enfermagem, 59(5), 680-683. doi: 10.1590/S003471672006000500016

Habhab-Rave, S. (2010). The role of the communities of practice in the process of knowledge management in innovative enterprises: a case study through intersite comparison. Revue des sciences de gestion, 45(241), 43-54. doi: 10.1590/S1415-65552012000600004

Herrmann, T. (2009). Socio-technical appropriation of web 2.0 for continuing. CSCW $2009-$ Workshop 3.0. Recuperado de www.sociotech-lit.de/Herr08-SAo.pdf

Johnson, S. (2001). Emergence: the connected lives of ants, brains, cities and software. London: Penguin Books. 
Kolfschoten, G. L., Niederman, F., Briggs, R. O., \& Vreede, G.-J. de (2012). Facilitation roles and responsibilities for sustained collaboration support in organizations. Journal of Management Information Systems, 28(4), 129-162. doi: 10.2753/MIS0742-1222280406

Laboratório de Estudos em Inteligência Coletiva e Biopolítica. (2008, June 13). Planilha dos 4c. Recuperado em http://www.linc.org.br/?p=122

Lévy, P. (1994). A inteligência coletiva: por uma antropologia do ciberespaço. São Paulo: Loyola.

Maria, A. S. R. I., Faria, V. C. M., \& Amorim, M. A. (2008). A comunidade de prática da rede nós: colaborando e compartilhando conhecimentos em arranjos produtivos locais. Organização \& Sociedade, 15(44), 149-170.

Moeckel, A., \& Forcellini, F. A. (2007). Collaborative product pre-development: an architecture proposal. In G. Loureiro \& R. Curran (Orgs.), Complex systems concurrent engineering: collaboration, technology innovation and sustainability (pp. 471-478). London: Springer.

Morgan, G., \& Smircich, L. (1980). The case for qualitative research. Academy of Management Review, 5(4), 491-500. doi: 10.5465/AMR.1980.4288947

Nonaka, I., \& Takeuchi, H. (1997). Criação de conhecimento na empresa: como as empresas japonesas geram a dinâmica da inovação. Rio de Janeiro: Campus.

Primo, A. (2003). Quão interativo é o hipertexto? da interface potencial à escrita coletiva. Fronteiras: Estudos Midiáticos, 5(2), 125-142.

Rheingold, H. (1996). A comunidade virtual. Lisboa: Gradiva.

Rheingold, H. (2002). Smart mobs: the next social revolution. Secaucus, NJ, USA: Perseus.

Ribeiro, J. C. M. de (2005). A gestão das informações nas redes informais: um desafio à tecnologia da informação. Focus: comunicação, cultura e conhecimento, 1(1), 9-14.

Robbins, S. P. (2013). Fundamentos do comportamento organizacional. São Paulo: Pearson.

Romani, C. C., \& Kuklinski, H. P. (2007). Planeta web 2.0: inteligencia colectiva o medios fast food. Recuperado http://books.google.com.br/books?id=ptMCLfJTSxEC\&printsec=frontcover\#v=onepage \&q\&f= false

Schommer, P. C. (2005). Comunidades de prática e articulação de saberes na relação entre universidade e sociedade (Tese de doutorado). Fundação Getúlio Vargas, São Paulo, SP, Brasil.

Serviço Brasileiro de Apoio às Micro e Pequenas Empresas. (2009). Associações. Recuperado de http://bis.sebrae.com.br/GestorRepositorio/ARQUIVOS_CHRONUS/bds/bds.nsf/DAD2C8C4D 5F6C26B8325766A005102D0/\$File/NT00042C26.pdf

Simpson, D. (2011). Reform, inequalities of process and the transformative potential of communities of practice in the pre-school sector of England. British Journal of Sociology of Education, 32(5), 699-716. doi: 10.1080/01425692.2011.596366

Souza-Silva, J. C. (2009). Condições e desafios ao surgimento de comunidades de práticas em organizações. Revista de Administração de Empresas, 49(2), 176-189. doi: 10.1590/S003475902009000200005

Souza-Silva, J. C., \& Davel, E. (2007). Da ação à colaboração reflexiva em comunidades de prática. Revista de Administração de Empresas, 47(3), 53-65. doi: 10.1590/S0034-75902007000300005 
Surowiecki, J. (2004). Cien major que uno: la sabiduria de la multidud o por qué la mayoria siempre es más inteligente que la minoria. Barcelona: Tendências.

Teixeira, R. R. (2005). O desempenho de um serviço de atenção primária à saúde na perspectiva da inteligência coletiva. Interface - Comunicação, Saúde, Educação, 9(17), 219-234. doi: 10.1590/S1414-32832005000200002

Turban, E., Aronson, J. E., \& Liang, T. P. (2005). Decision support systems and intelligent systems. Tualatin, OR, USA: Prentice Hall.

Xueguang, S. H. C. (2004). Research and analysis of the development of CSCW. Recuperado de http://en.cnki.com.cn/Article_en/CJFDTOTAL-JSGG200401001.htm

Wenger, E. (1998). Communities of practice: learning, meaning and identity. Cambridge: Cambridge University Press.

Wenger, E. (2001). Supporting communities of practices a survey of community-oriented technologies: how to make sense of this emerging market understand the potential of technology and set up a community platform. Version 1.3. Recuperado de http://archive2.nmc.org/projects/dkc/Technology_Survey.doc

Wenger, E. (2003). Communities of practice and social learning systems. In D. Nicolini, S. Gherardi, D. Yanow (Orgs.), Knowing in organizing: a practice-based approach (pp. 3-31). New York: M. E. Sharper.

Wenger, E., Mcdermott, R., \& Snyder, W. M. (2002). Cultivating communities of practice. Boston: Harvard Business School Press.

Wenger, E., White, N., \& Smith, J. D. (2009). Digital habitats: stewarding technology for communities. Portland: CPsquare.

Wenger, E., White, N., \& Smith, J. D. (n. d.). Technology for communities project. Recuperado de http://cpsquare.org/wiki/Technology_for_Communities_project

Woodward, R. (2011, March 14). Benefits of groupware for collaborative teams. Recuperado de http://www.brighthub.com/office/collaboration/articles/110152.aspx

Yin, R. K. (2010). Estudo de caso: planejamento e métodos (4a ed.). Porto Alegre: Bookman. 